\title{
INSTRUMENTO DE AUTOEVALUACIÓN DE LOS PROGRAMAS DE FORMACIÓN VIRTUAL A PARTIR DE CRITERIOS BÁSICOS DE CALIDAD
}

\author{
SELF-ASSESSMENT TOOL FOR VIRTUAL TRAINING PROGRAMS FROM BASIC \\ QUALITY STANDARDS
}

\author{
Albenis Cortés Rincón*
}

\section{RESUMEN}

Este documento presenta una propuesta de instrumento de autoevaluación que permita a los encargados de formación virtual hacer una revisión de su proceso a partir de criterios básicos de calidad en los programas de educación a distancia (EaD). El mismo es resultado del proyecto de investigación enmarcado en el tema de calidad de los programas de formación virtual adelantado por la Fundación Universitaria Panamericana. El proceso de construcción del instrumento se ubica en el paradigma cualitativo, de tipo exploratorio, al buscar indagar sobre el estado de la situación a partir de la revisión documental del tema. El instrumento consta de tres categorías claves en el establecimiento de criterios de calidad: 1) administrativas, 2) diseño curricular, desarrollo y evaluación de materiales y 3) profesorales.

Palabras clave: calidad en programas de formación a distancia, certificación, acreditación de calidad.

\begin{abstract}
This paper presents a proposal for a self-assessment tool that allows virtual training managers do a review of the process from basic criteria of quality in distance education programs (EaD). It is the result of a research project on the subject framed quality virtual training programs are advanced by the Fundación Universitaria Panamericana. The process of construction is based in the qualitative paradigm, exploratory, seeking to inquire about the status of the situation from the literature review of the topic. The instrument have three categories: 1) administrative, 2) curriculum design, development and evaluation of materials and 3) professorial.
\end{abstract}

Keywords: quality of distance education programs, certification, quality accreditation.

Fecha de recepción: Junio 09 de 2014 / Fecha de aceptación: Octubre 03 de 2014

Tipología: Artículo Corto

Para citar el artículo: Cortés, R. A. (2014). Instrumento de autoevaluación de los programas de formación virtual a partir de criterios básicos de calidad. Praxis, Vol. 10, 165 - 175

\footnotetext{
* Estudiante de Doctorado en Educación UAB (Barcelona, España). Magister en docencia. Especialista en Psicología educativa e Ingeniera de sistemas. Docente investigadora de la Fundación Universitaria Panamericana, España.E-mail: albeniscortes@unipanamericana.edu.co.
} 


\section{INTRODUCCIÓN}

$\mathrm{L}$ a presente propuesta surge desde la problemática asociada a la modalidad de aprendizaje virtual y expuesto en documentos presentados por Korniejczuk, (2003); Dávila (2006) y Fernández (2005), entre otros. La educación tiene el reto y la obligación social de ser mejor cada día y las instituciones encargadas de la misma tienen la obligación de brindar a sus estudiantes la seguridad de estar invirtiendo bien su tiempo y dinero.

Los programas de educación a distancia tienen mayor complejidad para cumplir las expectativas de sus estudiantes, Dentro del contexto académico de acuerdo con Korniejczuk (2003) son puntos de preocupación en la educación a distancia factores asociados a: 1) Uso responsable del ambiente de aprendizaje a distancia, 2) fraude o abuso del ambiente de aprendizaje, 3) confianza en los procesos de acreditación de calidad de los ambientes de educación a distancia, 4) necesidades de generar nuevas formas de evaluar la calidad, 5) tranquilidad del gobierno frente al principio de autorregulación de las IES, 6) características distintivas de la oferta educativa a distancia dentro de los campus tradicionales o distribuidos por los nuevos proveedores.

Es evidente la complejidad en el proceso de seguimiento de los programas de educación a distancia EaD y es así como Dávila (2006) establece siete barreras para el aseguramiento de la calidad: 1) legales, se refiere a la reglamentación, en cuanto al tema de acreditación de programas virtuales, 2) profesorales, tiene que ver con la falta de capacitación y de incentivos proporcionados a los docentes, 3) estudiantiles, no hay autodisciplina para asumir esta modalidad de estudio, 4) administrativas, falta de organización y mejoramiento continuo de los proyectos de educación a distancia, 5) resistencia, hace referencia a la poca apropiación de las universidades de orden tradicional frente a esta clase de modalidad educativa, 6) metodológicas, planeación, diseño curricular y evaluación deficientes, además de métodos poco funcionales en el proceso de enseñanza-aprendizaje y 7) tecnológicas, hace referencia al poco acceso y apropiación de las tecnologías en varias poblaciones para acceder a esta modalidad de estudio.

Las barreras antes mencionadas generan en los estudiantes inconformismo una vez ha establecido sus expectativas de formación académica y esto desencadena en muchos casos en la deserción escolar y genera una imagen negativa de la modalidad. De allí la importancia de establecer una relación clara entre la calidad esperada, la calidad percibida, y la calidad realizada. En el estudio adelantado por la European Training Village (citado por Fernández, 2005) sobre la situación de la calidad en el e-learning realizado con 433 profesionales procedentes de diversos sectores vinculados con la enseñanza en Europa, el $61 \%$ de los encuestados calificó como baja o insuficiente la calidad de los programas y solo el $1 \%$ estimó que los programas de e-learning podían considerarse como excelentes.

La presente propuesta tiene como objetivo diseñar un instrumento de autoevaluación que permita a los responsables de los programas de formación virtual identificar puntos neurálgicos del proceso a partir de criterios básicos de calidad en la línea del autoaprendizaje que conduzca a la revisión y posterior acreditación de los programas. La Comisión de Acreditación de la Calidad de la Educación (EQAC) (2003) establece que la autoevaluación y la evaluación de pares es la base de la acreditación de calidad de la educación superior, estableciendo tres fases: autoaprendizaje, revisión y acreditación.

\section{¿Por qué es importante la calidad en la educación?}

Organizaciones como la UNESCO y el Banco Mundial generan dinámicas globales en torno a la calidad de la educación. América Latina y el Caribe no son ajenas a esa situación y por ello han trabajado en el tema de evaluación y acreditación de los programas universitarios. La 
acreditación implica satisfacer la demanda de los usuarios y dar oportunidades de crecimiento a los países, base del desarrollo humano. Estas dinámicas hacen que día a día se reevalúen el propósito y las metodologías para lograr un mayor impacto y cubrir todas las necesidades propias para hacer frente en un mundo competitivo y en constante evolución, (Àguila, 2004). Aunado a esto surge la "necesidad de mejorar la calidad de la evaluación y adoptar procedimientos estandarizados, aumentar el reconocimiento externo del valor de la acreditación y atender al creciente fenómeno de la educación transnacional” (Sebastian, 2008, p. 4).

Hasta comienzos de los años 60 del siglo XX, la visión tradicional y estática de la calidad de la educación universitaria se basaba en cuatro principios: a) tradición de la institución, b) exclusividad de profesores, c) calidad de los alumnos e d) implementación de recursos materiales. Los años de escolaridad reflejaban la calidad de los egresados; en el siglo XXI estas dinámicas dinámicas han sido transformadas y han surgido nuevas necesidades de cambio asociados a recursos y métodos obsoletos y el surgimiento de nuevas instituciones universitarias, lo que provoca el fin del monopolio y da paso a la competencia en en pro del estudiante (Aguila, 2005).

La presenta propuesta se fundamenta en conceptos claves para su comprensión: calidad en programas de formación a distancia, certificación, acreditación de calidad, así:

Calidad en programas de formación a distancia: La norma ISO 9000:2000 (Colin, 2000) establece como primer principio, la organización orientada al cliente, lo cual implica comprender las necesidades actuales y futuras de los clientes con el fin de "satisfacer sus requerimientos y esforzarse por superar sus expectativas” (p.183); de allí que se deben articular los programas de formación con la realidad del mundo, de la nación, de la región, de la comunidad y de las expectativas del estudiante.
Es responsabilidad de las instituciones educativas borrar la barrera que puede llegar a existir entre los profesionales graduados de los programas tradicionales presenciales y los programas virtuales. Las instituciones educativas deben ofrecer a sus estudiantes la oportunidad de tener acceso a la educación virtual de calidad, que se ajuste a las necesidades individuales de cada uno de los estudiantes y garantice su futura participación activa en la sociedad.

La calidad entendida como ausencia de deficiencias reduce a su mínima expresión la generación de errores lo cual impacta positivamente al proceso reduciendo las fallas de operación y aumentando la satisfacción del cliente (Dávila, 2006). Las instituciones deben estar alerta ante las posibles falencias, de esta forma se reduce el inconformismo por parte del estudiante sobre el tipo de formación que se le está brindando y se reducen los costos asociados a los reprocesos.

García (2002) plantea como uno de los problemas referidos a la integración de las tecnologías en los procesos de educación a distancia, "la falta de capacidad de las instituciones para transformar y actualizar su estructura organizativa, sus procesos de aprendizaje y sus programas de reclutamiento, formación y actualización de profesores, investigadores, directivos y administrativos” (p.17). Dávila (2006) plantea la importancia de ejercer control y acompañamiento a los diversos procedimientos asociados a "la gestión académica, gestión administrativa, modelo educativo, producción de materiales educativos, infraestructura tecnológica, servicios estudiantiles, políticas y reglamentaciones internas, y desarrollo de una cultura de innovación e investigación” (p.3).

Certificación en EaD: Hace referencia al "resultado de un proceso por el que se verifica y documenta el cumplimiento de requisitos de calidad previamente establecidos, puede referirse a procesos o personas" (Riaces, 2004, p. 22). De esta forma el certificar la calidad de los 
programas de formación a distancia garantiza la mejora de las instituciones, brindando el reconocimiento ante las autoridades competentes y ante su competencia con el fin de lograr un alto posicionamiento.

Acreditación de Calidad para la Educación a Distancia (EaD): El Distance Education and Training Council DETC (2009) establece la acreditación como el principal medio de control de calidad en la educación superior de los Estados Unidos y un recurso importante para la mejora de la calidad. La acreditación se ha asociado con juicios de pares sobre la calidad y ha sido el trabajo de las agencias de acreditación no gubernamentales como DETC.

El Consejo Nacional de Acreditación (CNA, 2013) establece la acreditación como "un instrumento para promover y reconocer la dinámica del mejoramiento de la calidad y para precisar metas de desarrollo institucional; por consiguiente, implica la promoción de la cultura de la calidad en las instituciones y, por ende, la generación de sistemas de evaluación permanente y de mejoramiento continuo" (p. 7). Lo anteriormente expuesto proporciona una idea de cómo mediante la acreditación se establece un proceso de mejora continua en los procesos educativos, que beneficia a las instituciones, pero principalmente a los estudiantes que acceden a sus programas. De esa manera, se fomenta la competencia de las instituciones educativas para ofrecer a sus estudiantes procesos de formación con altos indicadores de calidad que les permita aumentar su nivel y destacarse frente a otras instituciones que puedan ofrecer los mismos servicios.

\section{METODOLOGÍA}

El proceso de construcción del instrumento se ubica en el paradigma cualitativo, de tipo exploratorio, al buscar indagar sobre el estado de la situación a partir de la revisión documental sobre el tema.

\section{RESULTADOS}

\section{Instrumento de autoevaluación}

El presente instrumento de autoevaluación presenta criterios básicos de calidad que deben cumplir los programas de formación virtual, asociados a tres categorías: 1) administrativas, 2) diseño curricular, desarrollo y evaluación de materiales y 3) profesorales (tabla 1).

\section{Tabla 1}

Distribución de criterios de evaluación por categoría

\begin{tabular}{|c|l|}
\hline \multicolumn{2}{|c|}{ CATEGORÍA } \\
\hline 1 & ADMINISTRATIVAS \\
\hline 1.1 & Aspectos generales \\
\hline 1.2 & Tiempos de dedicación \\
\hline 1.3 & Asociación con otras entidades \\
\hline 2 & $\begin{array}{l}\text { DISEÑO CURRICULAR, DESARROLLO Y } \\
\text { EVALUACIÓN DE MATERIALES }\end{array}$ \\
\hline 2.1 & Diseño curricular \\
\hline 2.2 & Desarrollo y evaluación de materiales \\
\hline 3 & PROFESORALES \\
\hline 3.1 & Formación de los formadores \\
\hline 3.2 & Investigación \\
\hline
\end{tabular}

Fuente: Elaboración propia

La escala de valoración (tabla 2), considera 5 niveles que abordan desde la no existencia del criterio hasta un estado de mejoramiento continuo adoptado por la institución educativa. 
Tabla 2

Escala de valoración

\begin{tabular}{|l|l|l|}
\hline \multicolumn{2}{|l|}{ Nivel 4} & $\begin{array}{l}\text { Mejoramiento continuo: Los pro- } \\
\text { gramas de formación virtual han } \\
\text { alcanzado un nivel de desarrollo } \\
\text { que ya involucra la lógica del me- } \\
\text { joramiento continuo, puesto que } \\
\text { evalúa sus procesos y, en conse- } \\
\text { cuencia, los ajusta y mejora. }\end{array}$ \\
\hline Nivel 3 & $\begin{array}{l}\text { Apropiación: El estado de la institución se } \\
\text { caracteriza por la implementación del as- } \\
\text { pecto, es decir, hay evidencia de su nivel de } \\
\text { desarrollo, ha sido difundido ampliamente y } \\
\text { es reconocido por la comunidad educativa. }\end{array}$ \\
\hline Nivel 2 & $\begin{array}{l}\text { Pertinencia: El estado de los programas de formación } \\
\text { responde a dos condiciones básicas: los retos insti- } \\
\text { tucionales (articulación al PEI y a las necesidades de } \\
\text { los usuarios) y las demandas externas (normatividad } \\
\text { vigente y contexto). }\end{array}$ \\
\hline Nivel 1 & \multicolumn{3}{|l|}{$\begin{array}{l}\text { Existencia: El estado de los programas de formación se carac- } \\
\text { teriza por un desarrollo parcial. Se han considerado los criterios } \\
\text { pero las acciones adelantadas son insuficientes o no se tiene } \\
\text { evidencia de su existencia. }\end{array}$} \\
\hline Nivel 0 & $\begin{array}{l}\text { No existe: Los programas de formación virtual carecen de lineamientos } \\
\text { claros sobre el tema. }\end{array}$ \\
\hline
\end{tabular}

Fuente: Elaboración propia

El apartado de la categoría administrativa hace referencia a la organización y mejoramiento continuo de los proyectos de educación a distancia. Considera aspectos generales a nivel administrativo, tiempos de dedicación y asociación con otras entidades (tabla 3).

Tabla 3

Criterios de evaluación - categoría administrativa

\begin{tabular}{|c|c|c|c|c|c|c|}
\hline \multirow{2}{*}{$\#$} & \multirow{2}{*}{ CRITERIO DE EVALUACIÓN } & \multicolumn{5}{|c|}{ NIVEL } \\
\hline & & 0 & 1 & 2 & 3 & 4 \\
\hline \multicolumn{7}{|c|}{$\begin{array}{l}\text { 1.1. Aspectos generales. } \\
\text { Existen estrategias institucionales orientadas a ... }\end{array}$} \\
\hline 1 & Cumplir la misión, metas y objetivos. & & & & & \\
\hline 2 & $\begin{array}{l}\text { Identificar si la misión institucional se integra en el } \\
\text { trabajo de cada departamento o las unidades acadé- } \\
\text { micas y administrativas logrando alinear sus deberes } \\
\text { con la misión de la institución. }\end{array}$ & & & & & \\
\hline 3 & $\begin{array}{l}\text { Realizar mediciones y comparaciones con programas } \\
\text { que se ofrecen en otras instituciones debidamente } \\
\text { acreditadas. }\end{array}$ & & & & & \\
\hline 4 & $\begin{array}{l}\text { Generar un modelo de evaluación institucional vali- } \\
\text { dado para identificar su impacto. }\end{array}$ & & & & & \\
\hline
\end{tabular}




\begin{tabular}{|c|c|c|c|c|c|c|}
\hline \multirow{2}{*}{$\#$} & \multirow{2}{*}{ CRITERIO DE EVALUACIÓN } & \multicolumn{5}{|c|}{ NIVEL } \\
\hline & & 0 & 1 & 2 & 3 & 4 \\
\hline 5 & $\begin{array}{l}\text { Definir un perfil competencial del tutor virtual como } \\
\text { parte del proceso de selección y contratación. }\end{array}$ & & & & & \\
\hline 6 & $\begin{array}{l}\text { Definir criterios de selección dentro del proceso de } \\
\text { admisión de estudiantes. }\end{array}$ & & & & & \\
\hline 7 & $\begin{array}{l}\text { Evitar suplantación o fraude en el proceso de } \\
\text { formación. }\end{array}$ & & & & & \\
\hline 8 & $\begin{array}{l}\text { Generar un plan de seguimiento a los egresados de } \\
\text { los programas de formación virtual. }\end{array}$ & & & & & \\
\hline 9 & $\begin{array}{l}\text { Acompañar a estudiantes y tutores frente a inquietu- } \\
\text { des de tipo administrativo. }\end{array}$ & & & & & \\
\hline 10 & $\begin{array}{l}\text { Garantizar la consecución de los objetivos de } \\
\text { aprendizaje. }\end{array}$ & & & & & \\
\hline 11 & $\begin{array}{l}\text { Realizar cambios o modificaciones en la estructura } \\
\text { curricular de los programas de formación virtual. }\end{array}$ & & & & & \\
\hline 12 & $\begin{array}{l}\text { Desarrollar planes de mantenimiento y mejora de } \\
\text { laboratorios remotos, plataformas de aprendizaje y } \\
\text { espacios alternos de formación. }\end{array}$ & & & & & \\
\hline \multicolumn{7}{|c|}{$\begin{array}{l}\text { 1.2. Tiempos de dedicación } \\
\text { Estrategias que garanticen... }\end{array}$} \\
\hline 13 & $\begin{array}{l}\text { Horas efectivas de dedicación en plataforma por parte } \\
\text { de los estudiantes. }\end{array}$ & & & & & \\
\hline 14 & $\begin{array}{l}\text { Tiempos de dedicación fuera de plataforma por parte } \\
\text { de los estudiantes. }\end{array}$ & & & & & \\
\hline 15 & $\begin{array}{l}\text { Participación periódica de los estudiantes en el desa- } \\
\text { rrollo de las actividades de aprendizaje. }\end{array}$ & & & & & \\
\hline 16 & $\begin{array}{l}\text { Identificar problemas en relación con el tiempo de } \\
\text { dedicación por parte de los estudiantes. }\end{array}$ & & & & & \\
\hline 17 & $\begin{array}{l}\text { Informar los tiempos de dedicación de las asignaturas } \\
\text { previo a la inscripción de créditos académicos. }\end{array}$ & & & & & \\
\hline 18 & $\begin{array}{l}\text { Horas efectivas de dedicación en plataforma por parte } \\
\text { de los tutores. }\end{array}$ & & & & & \\
\hline 19 & $\begin{array}{l}\text { Retroalimentación pertinente en el tiempo por parte } \\
\text { de los tutores frente a las actividades de aprendizaje. }\end{array}$ & & & & & \\
\hline 20 & $\begin{array}{l}\text { Cálculo y validación de los tiempos de dedicación } \\
\text { por parte de los docentes frente al número de horas } \\
\text { reconocidas en la asignación salarial. }\end{array}$ & & & & & \\
\hline 21 & $\begin{array}{l}\text { Asignación de tiempo docente para la planeación y } \\
\text { actualización de las asignaturas que se imparten. }\end{array}$ & & & & & \\
\hline \multicolumn{7}{|c|}{ 1.3. Asociación con otras entidades } \\
\hline 22 & $\begin{array}{l}\text { Existencia de por lo menos una entidad externa que } \\
\text { supervise los programas de formación ofertados. }\end{array}$ & & & & & \\
\hline \multirow[t]{2}{*}{23} & $\begin{array}{l}\text { Asociación con otras entidades. Establecimiento de } \\
\text { redes de conocimiento y alianzas estratégicas. }\end{array}$ & & & & & \\
\hline & Total & & & & & \\
\hline
\end{tabular}

Fuente: Elaboración propia 
El apartado diseño curricular, desarrollo y evaluación materiales hace referencia a los métodos empleados en el proceso de enseñanza-aprendizaje, planeación de los programas, desarrollo de materiales, derechos de autor y aspectos asociados a la evaluación de materiales para validar su aplicabilidad y pertinencia (tabla 4).

Tabla 4

Criterios de evaluación - categoría diseño curricular, desarrollo y evaluación materiales

\begin{tabular}{|c|c|c|c|c|c|c|}
\hline \multirow{2}{*}{ \# } & \multirow{2}{*}{ CRITERIO DE EVALUACIÓN } & \multicolumn{5}{|c|}{ NIVEL } \\
\hline & & 0 & 1 & 2 & 3 & 4 \\
\hline \multicolumn{7}{|c|}{ 2.1. DISEÑO CURRICULAR } \\
\hline 1 & $\begin{array}{l}\text { Los programas son diseñados considerando el } \\
\text { desarrollo de competencias específicas para } \\
\text { desempeñarse en la sociedad actual. }\end{array}$ & & & & & \\
\hline 2 & $\begin{array}{l}\text { Existen estrategias o temáticas que permitan el } \\
\text { desarrollo de habilidades profesional aplicables } \\
\text { en el campo laboral }\end{array}$ & & & & & \\
\hline 3 & $\begin{array}{l}\text { Las asignaturas cuentan con una estructura } \\
\text { definida. }\end{array}$ & & & & & \\
\hline 4 & $\begin{array}{l}\text { Las asignaturas son diseñadas a partir de acti- } \\
\text { vidades prácticas. }\end{array}$ & & & & & \\
\hline 5 & $\begin{array}{l}\text { Las habilidades técnicas son desarrolladas a } \\
\text { través de laboratorios de práctica. }\end{array}$ & & & & & \\
\hline 6 & $\begin{array}{l}\text { Las asignaturas cuentan con actividades teóricas } \\
\text { orientadas a la adquisición de conocimientos. }\end{array}$ & & & & & \\
\hline 7 & Se consideran ritmos diferentes de aprendizaje. & & & & & \\
\hline 8 & $\begin{array}{l}\text { Cada una de las actividades de aprendizaje es- } \\
\text { tablece tiempos y tareas claramente definidas. }\end{array}$ & & & & & \\
\hline 9 & $\begin{array}{l}\text { Estrategias apoyadas en las TIC para aumentar } \\
\text { la calidad de la enseñanza. }\end{array}$ & & & & & \\
\hline 10 & $\begin{array}{l}\text { Desarrollo de investigaciones o estudios que } \\
\text { permitan identificar el impacto que tiene la } \\
\text { incorporación de TIC en los procesos de forma- } \\
\text { ción virtual }\end{array}$ & & & & & \\
\hline 11 & $\begin{array}{l}\text { Procedimiento para realizar el cálculo de los } \\
\text { tiempos de dedicación en cada una de las } \\
\text { asignaturas. }\end{array}$ & & & & & \\
\hline 12 & $\begin{array}{l}\text { Procedimiento para definir los objetivos de } \\
\text { enseñanza-aprendizaje }\end{array}$ & & & & & \\
\hline 13 & $\begin{array}{l}\text { Estrategias de seguimiento que permitan } \\
\text { identificar si las metodologías se encuentran } \\
\text { enmarcadas dentro del modelo pedagógico ins- } \\
\text { titucional. }\end{array}$ & & & & & \\
\hline 14 & $\begin{array}{l}\text { Existencia de procedimientos que permitan vali- } \\
\text { dar la efectividad de las estrategias metodologías } \\
\text { aplicadas. }\end{array}$ & & & & & \\
\hline
\end{tabular}




\begin{tabular}{|c|c|c|c|c|c|c|}
\hline \multirow{2}{*}{$\#$} & \multirow{2}{*}{ CRITERIO DE EVALUACIÓN } & \multicolumn{5}{|c|}{ NIVEL } \\
\hline & & 0 & 1 & 2 & 3 & 4 \\
\hline 15 & $\begin{array}{l}\text { Estrategias de acompañamiento a las iniciativas } \\
\text { de desarrollo educativo por parte de estudiantes } \\
\text { y docentes. }\end{array}$ & & & & & \\
\hline 16 & $\begin{array}{l}\text { Estrategias que promuevan la interacción entre } \\
\text { docentes y estudiantes más allá de las asignatu- } \\
\text { ras que se imparten con la creación de proyectos } \\
\text { interdisciplinares o actividades de bienestar } \\
\text { universitario. }\end{array}$ & & & & & \\
\hline \multicolumn{7}{|c|}{ 2.2. DESARROLLO Y EVALUACIÓN DE MATERIALES } \\
\hline 17 & $\begin{array}{l}\text { Estrategias de promoción para el desarrollo de } \\
\text { nuevos materiales y recursos educativos. }\end{array}$ & & & & & \\
\hline 18 & $\begin{array}{l}\text { Asignación de tiempo docente a la actualiza- } \\
\text { ción y creación de actividades y materiales } \\
\text { educativos. }\end{array}$ & & & & & \\
\hline 19 & $\begin{array}{l}\text { Existencia y uso de los repositorios de objetos } \\
\text { de aprendizaje y material académico. }\end{array}$ & & & & & \\
\hline 20 & $\begin{array}{l}\text { Existencia de centros de apoyo al docente para } \\
\text { el desarrollo de nuevos materiales con acompa- } \\
\text { ñamiento sistemático de diseñadores formativos. }\end{array}$ & & & & & \\
\hline 21 & $\begin{array}{l}\text { Identificación y documentación de aciertos y } \\
\text { dificultades asociadas al desarrollo de material } \\
\text { educativo. }\end{array}$ & & & & & \\
\hline 22 & $\begin{array}{l}\text { Los materiales son desarrollados en su totalidad } \\
\text { con recursos propios o sobre los que se tenga los } \\
\text { derechos de uso, reproducción y distribución. }\end{array}$ & & & & & \\
\hline 23 & $\begin{array}{l}\text { Establecimiento de políticas institucionales cla- } \\
\text { ras relacionadas con la publicación de material } \\
\text { al interior de los cursos. }\end{array}$ & & & & & \\
\hline 24 & $\begin{array}{l}\text { Se hacen verificaciones a lo publicado en plata- } \\
\text { forma con el fin de garantizar la correcta citación } \\
\text { y uso de materiales sobre los cuales no se tiene } \\
\text { derecho de publicación, reproducción y uso. }\end{array}$ & & & & & \\
\hline 25 & $\begin{array}{l}\text { Validación de los objetivos de enseñanza-apren- } \\
\text { dizaje establecidos para el programa. }\end{array}$ & & & & & \\
\hline 26 & $\begin{array}{l}\text { Establecimiento de parámetros de medición que } \\
\text { permitan evidenciar que los objetivos del pro- } \\
\text { grama son alcanzables a través de las estrategias } \\
\text { metodológicas propuestas. }\end{array}$ & & & & & \\
\hline 27 & $\begin{array}{l}\text { Existencia de criterios de evaluación y promo- } \\
\text { ción en cada una de las asignaturas. }\end{array}$ & & & & & \\
\hline 28 & $\begin{array}{l}\text { Validación y pertinencia de los materiales pu- } \\
\text { blicados al interior de los cursos orientados al } \\
\text { logro de los objetivos de formación propuestos. }\end{array}$ & & & & & \\
\hline 29 & $\begin{array}{l}\text { Pruebas piloto de los cursos a través de expertos } \\
\text { en contenido y docentes para validar la pertinen- } \\
\text { cia disciplinar. }\end{array}$ & & & & & \\
\hline
\end{tabular}




\begin{tabular}{|c|l|l|l|l|l|l|}
\hline \multirow{2}{*}{$\#$} & \multicolumn{2}{|c|}{ CRITERIO DE EVALUACIÓN } & \multicolumn{5}{|c|}{ NIVEL } \\
\cline { 3 - 7 } 30 & $\begin{array}{l}\text { Pruebas piloto con expertos metodológicos, tu- } \\
\text { tores y estudiantes, para validar la claridad de } \\
\text { las instrucciones y la adecuada navegación de } \\
\text { los contenidos. }\end{array}$ & & & & & \\
\hline 31 & $\begin{array}{l}\text { Seguimiento a la planeación de los contenidos, } \\
\text { temas y actividades presentes en los cursos. }\end{array}$ & & & & & \\
\hline & TOTAL & & & & & \\
\hline
\end{tabular}

Fuente: Elaboración propia

El apartado profesorales tiene que ver con la capacitación e incentivos proporcionados a los docentes, así como el fomento de temas relacionados con la investigación, (tabla 5).

\section{Tabla 5}

Criterios de evaluación - categoría profesorales

\begin{tabular}{|c|c|c|c|c|c|c|}
\hline \multirow{2}{*}{ \# } & \multirow{2}{*}{ CRITERIO DE EVALUACIÓN } & \multicolumn{5}{|c|}{ NIVEL } \\
\hline & & 0 & 1 & 2 & 3 & 4 \\
\hline \multicolumn{7}{|c|}{ 3.1. FORMACIÓN DE LOS FORMADORES } \\
\hline 1 & Destinación de recursos para la formación de docentes. & & & & & \\
\hline 2 & $\begin{array}{l}\text { Existencia de centros de apoyo que faciliten el trabajo } \\
\text { que desarrollan los docentes. }\end{array}$ & & & & & \\
\hline 3 & $\begin{array}{l}\text { Existencia de programas de actualización en temas } \\
\text { específicos de las disciplinas. }\end{array}$ & & & & & \\
\hline 4 & $\begin{array}{l}\text { Participación de los docentes en la validación de las } \\
\text { competencias desarrolladas en los programas y perti- } \\
\text { nencia de las mismas. }\end{array}$ & & & & & \\
\hline 5 & $\begin{array}{l}\text { Implementación de programas de formación en uso e } \\
\text { incorporación de las TIC en los programas de forma- } \\
\text { ción virtual }\end{array}$ & & & & & \\
\hline 6 & $\begin{array}{l}\text { Estrategias orientadas a la formación de los formadores } \\
\text { a partir del perfil competencial de un tutor virtual. . }\end{array}$ & & & & & \\
\hline \multicolumn{7}{|c|}{ 3.2. INVESTIGACIÓN } \\
\hline 7 & $\begin{array}{l}\text { Promoción de la investigación por parte de los } \\
\text { docentes. }\end{array}$ & & & & & \\
\hline 8 & $\begin{array}{l}\text { Destinación de recursos para la investigación en pro- } \\
\text { gramas de formación virtual. }\end{array}$ & & & & & \\
\hline 9 & $\begin{array}{l}\text { Trabajo mancomunado entre docentes y estudiantes } \\
\text { en la creación de proyectos de investigación. }\end{array}$ & & & & & \\
\hline 10 & Proyecto de investigación con la comunidad. & & & & & \\
\hline
\end{tabular}




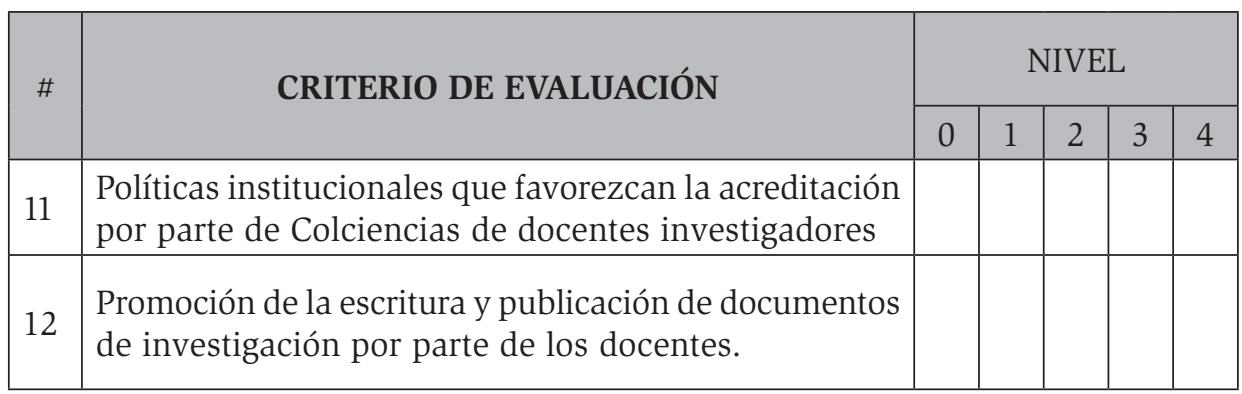

Fuente: Elaboración propia

\section{DISCUSIÓN}

Al ser consideradas únicamente 3 de las 7 categorías que se pueden establecer a partir de las barreras para el aseguramiento de la calidad establecidos por Dávila (2006), se hace necesario abordar en próximas etapas del proyecto temas asociados a: 1) Aspectos legales, se refiere a la reglamentación, en cuanto al tema de acreditación de programas virtuales, 2) Estudiantiles, no hay autodisciplina para asumir esta modalidad de estudio, 3) Resistencia, hace referencia a la poca apropiación de las universidades de orden tradicional frente a esta clase de modalidad educativa y 4) Tecnológicas, hace referencia al poco acceso y apropiación de las tecnologías en varias poblaciones, para así acceder a esta modalidad de estudio.

El presente instrumento se encuentra en etapa de implementación.

\section{AGRADECIMIENTOS}

Esta propuesta no hubiese sido posible sin la colaboración en calidad de asistentes de las estudiantes de la Facultad de Educación de la Fundación Universitaria Panamericana y hoy en día licenciadas Luz Yadira Gómez y Sonia Margarita Cruz Ortiz, quienes contribuyeron en la revisión bibliográfica y generación de las primeras versiones del instrumento.

\section{REFERENCIAS BIBLIOGRÁFICAS}

Águila, V. (2005). El concepto calidad en la educación universitaria: clave para el logro de la competitividad institucional. Revista Iberoamericana de Educación OEI. Número 35/5. 25-03-05, 1-7.

Comisión Nacional de Acreditación CNA. (2013). Lineamientos para la acreditación de programas de pregrado. Bogotá D.C.: Consejo Nacional de Acreditación CNA.

Colin, L. (2000). Las normas ISO 9000:2000 de Sistemas de Gestión de la Calidad. México: IEEE.

Dávila, A. (2006). Conclusiones del taller latinoamericano de reflexión sobre aseguramiento de la calidad de e-learning y educación a distancia. Congreso Iberoamericano de educación a distancia, (págs. 2-9). Barquisimeto, Venezuela.

Distance Education and Training Council DETC. (2009). Applying For Accreditation. Obtenido de http://www.detc.org/Seeking-Accreditation/Applying-forAccreditation.aspx 
Education Quality Accreditation Commission EQAC. (2013). Acreditación de instituciones educativas. Obtenido de http://www.acreditacion.info/index.html

Fernandez, V. (2005). E-aprendizaje en bibliotecología: perspectivas globales. En F. F. Martínez Arellano, Calidad y e-learning (págs. 74-91). México D.F., México: UNAM, Centro Universitario de Investigaciones Bibliotecológicas.

Korniejczuk, V. (2003). La acreditación de la educación superior presencial y a distancia en Estados Unidos y México. Revista de educación a distancia, 27-28-29.

MEN (2006). Guía No 11: Guía de Autoevaluación para el Mejoramiento Institucional. Bogotá D.C.:
Ministerio de Educación Nacional de Colombia. Obtenido de http://www.mineducacion.gov.co/ cvn/1665/article-91093.html

Riaces. (2004). Glosario Internacional RIACES de Evaluación de la Calidad y Acreditación. Madrid: Red Iberoamericana para la Acreditación de la Calidad de la Educación Superior.

Sebastian, J. (2008). Cooperación e internacionalización de las universidades. Buenos Aires: Editorial Biblos.

Garcia, L. (2002). Resistencias, cambio y buenas prácticas en la nueva educación a distancia. Revista iberoamericana de educación a distancia. Vol. 5 • n. ${ }^{\circ} 2$ diciembre, 2002 